Jour. d'Analyse Math., 80, (2000), 319-334.

\title{
A NEW APPROACH TO INVERSE SPECTRAL THEORY, III. SHORT-RANGE POTENTIALS
}

\author{
ALEXANDER RAMM ${ }^{1}$ AND BARRY SIMON ${ }^{2}$
}

\section{INTRODUCTION}

In this paper we discuss aspects of the spectral theory of half-line Schrödinger operators

$$
H u=-u^{\prime \prime}+q u,
$$

where $q \in L_{\text {loc }}^{1}([0, \infty))$. Indeed, all the $q$ 's considered in this paper will lie in $L^{1}([0, \infty), d x)$. The Weyl $m$-function $m(z)$ is defined for $\operatorname{Im} z>0$ by looking at the solution $u(x ; z)$ of $-u^{\prime \prime}+q u=z u$ which is $L^{2}$ at infinity (unique up to constants if $q$ is limit point and unique once a boundary condition at infinity is chosen if $q$ is limit circle) and setting

$$
m(z)=\frac{u^{\prime}(0 ; z)}{u(0 ; z)}
$$

In [10], we considered the fundamental object $A(\alpha)$ associated to $q$ by the relation

$$
m\left(-\kappa^{2}\right)=-\kappa-\int_{0}^{\infty} A(\alpha) e^{-2 \alpha \kappa} d \alpha,
$$

where $A(\alpha) \in L^{1}(0, a)$ for all $a$. Formula (1.3) holds in the sense of an absolutely convergent integral if $\operatorname{Re} \kappa$ is sufficiently large in case $q \in L^{1}$ ([10]) or if $q \in L^{\infty}([2])$. For general $q$, it holds in the same sense that a series is asymptotic to a function $([2],[10])$.

In [10], it is proven that if $q \in L^{1}(0, \infty)$, then

$$
|A(\alpha)-q(\alpha)| \leq\|q\|_{1}^{2} \exp \left(\alpha\|q\|_{1}\right) \text {. }
$$

\footnotetext{
${ }^{1}$ Mathematics Department, Kansas State University, Manhattan, KS 66506, USA. E-mail: ramm@math.ksu.edu

${ }^{2}$ Division of Physics, Mathematics, and Astronomy, 253-37, California Institute of Technology, Pasadena, CA 91125, USA. E-mail: bsimon@caltech.edu. This material is based upon work supported by the National Science Foundation under Grant No. DMS-9707661. The Government has certain rights in this material.

Date: May 11, 1999.

1991 Mathematics Subject Classification. Primary: 34B25, 34A55.

Key words and phrases. Weyl function, spectral theory, inverse problems.

To appear in J. d'Analyse Math.
} 
As explained in [10], $A$ is fundamental to an approach to inverse spectral theory. Our goal in this paper is to study $A$ as an interesting object in its own right and, in particular, using ideas implicit in Ramm [6] to obtain detailed information on the behavior of $A$ as $\alpha \rightarrow \infty$ when $q$ decays sufficiently fast as $x \rightarrow \infty$. Indeed, for potentials decaying rapidly enough, Ramm [6] stated the representation (1.3), but no proof was given (nor was there any connection of the function $A$ to the inverse problem for $q$. In [6] the inverse problem of finding the potential from the knowledge of the $m$-function has been solved for short-range potentials. A more detailed discussion of the result in [6] can be found in $[7])$.

Throughout this paper, we will suppose that

$$
\int_{0}^{\infty}(1+|x|)|q(x)| d x<\infty .
$$

More generally, we will consider for $n=0,1,2, \ldots, B \leq 0$ and $\ell \geq 0$, the space $C_{n}^{B, \ell}$ of all functions $f$ with $n-1$ classical derivatives and $f^{(n)} \in L^{1}(0, \infty)$ so that

$$
\int_{0}^{\infty}(1+|x|)^{\ell} e^{-B x}\left|q^{(j)}(x)\right| d x<\infty
$$

for $j=0,1, \ldots, n$. Thus (1.4) says $q \in C_{n=0}^{B=0, \ell=1}$.

Under condition (1.4), general principles (see, e.g., [5]) imply that for all $\kappa$ with $\operatorname{Re} \kappa \geq 0$, there is a unique solution $f(x, \kappa)$ of $-f^{\prime \prime}+$ $q f=-\kappa^{2} f$ so that $f(x, \kappa)=e^{-\kappa x}(1+o(1))$ as $x \rightarrow \infty$. We will set $f(\kappa) \equiv f(x=0, \kappa)$. Except for the change of variables $\kappa=-i k, f(x, \kappa)$ and $f(\kappa)$ are the standard Jost solution and Jost function. Both $f(x, \kappa)$ and $f(\kappa)$ are analytic in $\kappa$ in $\{\kappa \mid \operatorname{Re} \kappa>0\}$. If $q \in C_{n}^{B, \ell}$ for any $n, \ell$ and $B<0$, then $f(x, \kappa)$ and $f(\kappa)$ have analytic continuations into the region $\operatorname{Re} \kappa>B / 2$ (see Section 2 below).

It is easy to see and well known that [5]

(1) The zeros of $f$ in $\{\kappa \mid \operatorname{Re} \kappa>0\}$ occur precisely at those points $\kappa_{j}$ with $-\kappa_{j}^{2}$ a bound state of the operator $H$ with $u(0)=0$ boundary condition and each zero is simple.

(2) $f$ has no zeros on $\{\kappa \mid \operatorname{Re} \kappa=0, \kappa \neq 0\}$.

(3) If $f(0)=0$ and $q \in C_{n=0}^{B=0, \ell=2}$, then $f$ is $C^{1}$ and $f^{\prime}(0) \neq 0$. If $f(0)=0$, we say that $q$ has a zero energy resonance.

If $f$ can be analytically continued to $\{\kappa \mid \operatorname{Re} \kappa>B / 2\}$ for $B<0$, then zeros of $f$ in $\{\kappa \mid$ Re $\kappa<0\}$ are called resonances. They occur in complex conjugate pairs (since $f$ is real on the real axis). If $f^{\prime}\left(\kappa_{0}\right) \neq 0$ at a zero $\kappa_{0}$, we say that $\kappa_{0}$ is a simple resonance. Resonances need not be simple if $\operatorname{Re} \kappa_{0}<0$ although they are generically. 
The result stated in [6] can be phrased:

Theorem 1.1. Suppose that $q$ obeys (1.4) (lies in $C_{n=0}^{B=0, \ell=1}$ ) and does not have a zero energy resonance. Let $\left\{-\kappa_{j}^{2}\right\}_{j=1}^{J}$ be the negative eigenvalues of $H$ (with $u(0)=0$ boundary condition) with $\kappa_{j}>0$. Then

$$
A(\alpha)=\sum_{j=1}^{J} B_{j} e^{2 \alpha \kappa_{j}}+g(\alpha),
$$

where $g \in L^{1}(0, \infty)$. In particular, if $H$ has no bound states or zero energy resonance (e.g., if $q \geq 0)$, then $A \in L^{1}$.

Remarks. 1. The result stated in [6] assumes implicitly that there is no zero energy resonance. Details can be found in [7].

2. If $A \in L^{1}$, then (1.3) can be analytically continued to the entire region $\operatorname{Re} \kappa \geq 0$.

3. If $u_{j}(x)$ is the eigenfunction for $H$, at energy $-\kappa_{j}^{2}$, normalized so that $\int_{0}^{\infty}\left|u_{j}(x)\right|^{2} d x=1$, then

$$
B_{j}=-\frac{\left|u_{j}^{\prime}(0)\right|^{2}}{\kappa_{j}} .
$$

This follows from the relation between $A$ and the spectral measure [2]

$$
A(\alpha)=-2 \int_{-\infty}^{\infty} \lambda^{-1 / 2} \sin (2 \alpha \sqrt{\lambda}) d \rho(\lambda)
$$

and the fact that $d \rho(\lambda) \uparrow(-\infty, 0)=\sum_{j=1}^{J}\left|u_{j}^{\prime}(0)\right|^{2} \delta\left(\lambda+\kappa_{j}^{2}\right) d \lambda$.

To handle zero energy resonances, one needs an extra two powers of decay (just as (1.4) says more or less that $|q(x)|$ is bounded by $O\left(x^{-2-\varepsilon}\right)$, the condition in the next theorem says that $|q(x)|$ is more or less $O\left(x^{-4-\varepsilon}\right)$ ):

Theorem 1.2. Let $q \in C_{n=0}^{B=0, \ell=3}$. Suppose that $H$ (with $u(0)=0$ boundary condition) has a zero energy resonance and negative eigenvalues at $\left\{-\kappa_{j}^{2}\right\}_{j=1}^{J}$ with $\kappa_{j}>0$. Then

$$
A(\alpha)=B_{0}+\sum_{j=1}^{J} B_{j} e^{2 \alpha \kappa_{j}}+g(\alpha),
$$

where $g \in L^{1}(0, \infty)$.

These results are special cases of

Theorem 1.3. Let $q \in C_{n}^{B=0, \ell}$ where $\ell \geq 1$ and, if $q$ has a zero energy resonance, then $\ell \geq 3$. Then (1.6) (resp. (1.8) if there is a zero energy resonance) holds where $g \in C_{n}^{B=0, \ell-1}$ (resp. $C_{n}^{B=0, \ell-3}$ ). 
Remark. If $n \geq 1$, then $g^{\prime} \in L^{1}$ so $g \rightarrow 0$ pointwise. In general, if $q$ is not continuous, then $g$ may not go to zero. For example, let $q(x)=\sum_{j=0}^{\infty} \chi_{\left[n, n+a_{n}\right]}(x)$ where $a_{n}=e^{-n^{2}}$ and $\chi_{[a, b]}$ is the characteristic function of $[a, b]$. Since $A(\alpha)-q(\alpha)$ is continuous, we are guaranteed that $A(\alpha)$ has jump discontinuities at $\alpha=n$ and so $\varlimsup \lim A(\alpha)-\underline{\lim } A(\alpha) \geq 1$ and thus, $\lim A(\alpha)$ cannot be zero (since it cannot exist). In this case, $A=g$ since there are no bound states or zero energy resonance.

For $B<0$, we will prove:

Theorem 1.4. Let $q \in C_{n}^{B, \ell=0}$ with $B<0$. Let $\tilde{B} \in(B, 0)$ and let $\left\{-\kappa_{j}^{2}\right\}_{j=1}^{J}$ with $\kappa_{j}>0$ be the negative eigenvalues, $\left\{\lambda_{j}\right\}_{j=1}^{M}$ with $\lambda_{j} \leq 0$ the real resonances (a.k.a. anti-bound states) and $\left\{\mu_{j} \pm i \nu_{j}\right\}_{j=1}^{N}$ the complex resonances with $\tilde{B} \leq \mu_{j}<0$ with $\nu_{j}>0$. Suppose each resonance is simple. Then for suitable $\left\{B_{j}\right\}_{j=1}^{J},\left\{C_{j}\right\}_{j=1}^{M},\left\{D_{j}\right\}_{j=1}^{N}$, $\left\{\theta_{j}\right\}_{j=1}^{N}$, we have that

$A(\alpha)=\sum_{j=1}^{J} B_{j} e^{2 \alpha \kappa_{j}}+\sum_{j=1}^{M} C_{j} e^{2 \alpha \lambda_{j}}+\sum_{j=1}^{N} D_{j} e^{2 \mu_{j} \alpha} \cos \left(2 \nu_{j} \alpha+\theta_{j}\right)+\tilde{g}(\alpha)$,

where $\tilde{g}(\alpha) \in C_{n}^{\tilde{B}, \ell=0}$. In particular, if $H$ has no negative eigenvalues, the rate of decay of $A(\alpha)$ is determined by the resonance with the least negative value of $\lambda$ or $\mu$.

In Section 4, we will discuss what happens when there are non-simple resonances.

We note that in the appendix, we present a result on principal ideals in the space of Laplace transforms (Corollary A.5) that may be of interest in its own right.

We thank F. Gesztesy for useful comments.

\section{The Levin-Marchenko Representation}

The key to the proof of Theorems 1.1-1.4 is the formula

$$
m\left(-\kappa^{2}\right)=\frac{\left.\frac{\partial f(x, \kappa)}{\partial x}\right|_{x=0}}{f(x=0, \kappa)}
$$

for the solution $f$ of

$$
-f^{\prime \prime}+q f=-\kappa^{2} f
$$

with

$$
f(x, \kappa)=e^{-\kappa x}(1+o(1))
$$


as $x \rightarrow+\infty$.

We use a basic Laplace transform representation for $f(x, \kappa)$ found by Levin [3] and developed especially by Marchenko [4],[5] and the theory of Laplace transforms discussed in the appendix.

The Levin-Marchenko representation says that given any $q \in C_{n=0}^{B=0, \ell=1}$ $=\left\{q\left|\int_{0}^{\infty} x\right| q(x) \mid d x<\infty\right\}$, there is a continuous function $K(x, y)$ defined on $\{(x, y) \mid 0 \leq x \leq y<\infty\}$ with $\int_{x}^{\infty}|K(x, y)| d y<\infty$ for each $x$ so that

$$
f(x, \kappa)=e^{-\kappa x}+\int_{x}^{\infty} K(x, y) e^{-\kappa y} d y
$$

Define

$$
\sigma(x)=\int_{x}^{\infty}|q(y)| d y
$$

so $\int_{0}^{\infty} x|q(x)| d x<\infty$ is equivalent to $\sigma \in L^{1}$. Then the following bound on $K$ is known ([5], Lemma 3.1.1):

$$
|K(x, y)| \leq C \sigma\left(\frac{x+y}{2}\right)
$$

for some constant $C$. Indeed, one can take $C=\frac{1}{2} \exp \left(\int_{0}^{\infty} \sigma(y) d y\right)$. Moreover, $K$ is absolutely continuous in each variable. Indeed, if $H(u, v)$ is defined in $\{(u, v) \mid 0 \leq v \leq u\}$ by

$$
\begin{aligned}
& H(u, v)=K(u-v, u+v) \\
& K(x, y)=H\left(\frac{1}{2}(x+y), \frac{1}{2}(y-x)\right),
\end{aligned}
$$

then $H$ obeys (see [5], proof of Lemma 3.1.2):

$$
\begin{aligned}
& \frac{\partial H}{\partial u}(u, v)=-\frac{1}{2} q(u)-\int_{0}^{v} q(u-\beta) H(u, \beta) d \beta \\
& \frac{\partial H}{\partial v}(u, v)=\int_{u}^{\infty} q(\alpha-v) H(\alpha, v) d \alpha .
\end{aligned}
$$

¿From these bounds and equations, we have by a straightforward estimate that:

Theorem 2.1. If $q \in C_{n}^{B, \ell}$ with $\ell \geq 1$ and $B \leq 0$, then

$$
\begin{aligned}
& \text { (i) } K(x=0, \cdot) \in C_{n+1}^{B, \ell-1} \\
& \text { (ii) } \frac{\partial K}{\partial x}(x=0, \cdot) \in C_{n}^{B, \ell-1} .
\end{aligned}
$$

Remark. In fact, if $B<0$, we can replace $\ell-1$ by $\ell$. 


\section{Proof of the Main Theorems}

Theorems 1.3 and 1.4 will follow from Theorem 2.1, formulas (2.1), (2.4), and the results in the appendix. Let

$$
F(\kappa) \equiv f(x=0, \kappa)=1+\int_{0}^{\infty} K(x=0, y) e^{-\kappa y} d y
$$

By Theorem 2.1, $F \in \mathfrak{A}_{n+1}^{B, \ell-1}$ with $F(\infty)=1$. The Banach algebra $\mathfrak{A}_{n}^{B, \ell}$ is discussed in the appendix. Thus, by Theorem A.8

$$
F(\kappa)^{-1}=\sum_{\ell=1}^{\tilde{J}} \frac{a_{\ell}}{\kappa-\tilde{\kappa}_{\ell}}+G(\kappa)
$$

where

(i) If $B<0,\left\{\kappa_{\ell}\right\}_{\ell=1}^{\tilde{J}}$ are the zeros of $F$ on $\operatorname{Re} \kappa>B$ and $G \in \mathfrak{A}_{n+1}^{\tilde{B}, \ell-1}$ for any $\tilde{B}>B$.

(ii) If $B=0$ and $F(0) \neq 0$, then $G \in \mathfrak{A}_{n+1}^{B=0, \ell-1}$.

(iii) If $B=0$ and $F(0)=0$, we need $\ell \geq 3$ and then $G \in \mathfrak{A}_{n+1}^{B=0, \ell-3}$.

Remarks. 1. For (i), we suppose all resonances are simple.

2. For (ii), (iii), by general principles, the zeros in $\operatorname{Re} \kappa \geq 0$ occur only for $\operatorname{Im} \kappa=0$. Moreover, each such zero is simple (see Lemma 3.1.6 of $[5])$.

Moreover, since $K$ is $C^{1}$ in $y$, by an integration by parts, $F(\kappa)=$ $1+K(0,0) / \kappa+O\left(1 / \kappa^{2}\right)$, and thus

$$
F(\kappa)^{-1}=1-\frac{K(0,0)}{\kappa}+O\left(\frac{1}{\kappa^{2}}\right) .
$$

Indeed, by Theorem A.7,

$$
\kappa F(\kappa)^{-1}=\kappa-K(0,0)+\sum_{\ell=1}^{\tilde{J}} \frac{a_{\ell} \kappa_{\ell}}{\kappa-\kappa_{\ell}}+\tilde{G}(\kappa),
$$

where $\tilde{G}$ is the same $\mathfrak{A}$ space as $G$, except that the number of derivatives is $n$, not $n+1$. Moreover, $\tilde{G}(\infty)=0$.

Let

$$
\begin{aligned}
H(\kappa) & \left.\equiv \frac{\partial}{\partial x} f(x, \kappa)\right|_{x=0}=-\kappa-K(0,0)-\left.\int_{0}^{\infty} \frac{\partial K}{\partial x}(0, y)\right|_{x=0} e^{-\kappa y} d y \\
& \equiv-\kappa+\tilde{H}(\kappa),
\end{aligned}
$$

where $\tilde{H}$ lies in $\mathfrak{A}_{n}^{B, \ell-1}$ and $\tilde{H}(\infty)=-K(0,0)$. 
Therefore, by (3.2),

$$
\begin{aligned}
m\left(-\kappa^{2}\right) & =-\kappa F(\kappa)^{-1}+\tilde{H}(\kappa) F(\kappa)^{-1} \\
& =-\kappa+\sum_{\ell=0}^{\tilde{J}} b_{\ell}\left(\kappa-\kappa_{\ell}\right)^{-1}+\tilde{M}(\kappa),
\end{aligned}
$$

where $\tilde{M}(\infty)=0$ (since the $K(0,0)$ terms cancel) and $\tilde{M} \in \mathfrak{A}_{n}^{\tilde{B}, \ell_{0}}$ with $\ell_{0}=\ell-3$ (if $B=0$ and $F(0)=0$ ), and otherwise $\ell_{0}=\ell-1$ and $\tilde{B}=0$ (if $B=0)$, and otherwise $\tilde{B} \in(B, 0)$. Since $\left(\kappa-\kappa_{\ell}\right)^{-1}=$ $2 \int_{0}^{\infty} e^{2 \kappa_{\ell} t} e^{-2 \kappa t} d t$ if $\operatorname{Re} \kappa>\operatorname{Re} \kappa_{\ell}$, Theorems 1.3 and 1.4 are proven.

\section{EXTENSIONS AND REMARKS}

It is only for simplicity that we supposed the resonances were simple. By iterating Theorem A.4 if $f \in \mathfrak{A}_{n}^{B, \ell}$ and $\operatorname{Re} z_{0}>B$ and $z_{0}$ is a zero of $F$ of order $j$, then $g(z)=F(z) /\left(z-z_{0}\right)^{j}$ is in $\mathfrak{A}_{n+j}^{B, \ell}$. Thus, if some zeros of $f(\kappa)$ are of order $j,(3.4)$ need only be modified so that there are terms

$$
\sum_{\ell=0}^{\tilde{J}} \sum_{j=1}^{\bar{\nu}} b_{\ell}^{j}\left(\kappa-\kappa_{\ell}\right)^{-j},
$$

where $\bar{\nu}=\max ($ order of zeros in the strip). Since

$$
\frac{1}{\left(\kappa-\kappa_{\ell}\right)^{j}}=\frac{2}{(j-1) !} \int_{0}^{\infty} \alpha^{j-1} e^{-2 \alpha \kappa} e^{2 \alpha \kappa_{\ell}} d \alpha,
$$

we obtain an explicit formula for $A$ like in Theorem 1.4, but $C_{j} e^{2 \alpha \lambda_{j}}$ is replaced by a polynomial in $\alpha$ times $e^{2 \alpha \lambda_{j}}$ and $D_{j} e^{2 \mu_{j} \alpha} \cos \left(2 \nu_{j} \alpha+\theta_{j}\right)$ is replaced by $\sum_{k=0}^{\tilde{\nu}} d_{j, k} \alpha^{k} e^{\nu, \alpha} \cos \left(2 \nu_{j} \alpha+\theta_{j}^{(k)}\right)$.

If $q \in \mathfrak{A}_{n=0}^{B}$ for all $B$, for example, $q$ is compactly supported, it is known that there are always infinitely many resonances (see [8], pp. 280-282, [9], [11]). Thus $A(\alpha)$ does not decay faster than exponentially in these cases. It is natural to conjecture that $A(\alpha)$ never decays faster than exponentially as $\alpha \rightarrow \infty$ for $q \not \equiv 0$.

\section{Appendix A. On the Theory of Laplace Transforms}

In this appendix, we present some basic facts about Laplace transforms of functions that we need in the paper itself. Given the vast literature on Banach algebras, it may be that these results are presented elsewhere, but since we know of no precise reference, we present what is needed here. 
Definition. A weight is a strictly positive function, $w(\alpha)$, on $[0, \infty)$ that obeys:

(a) $w(0)=1$.

(b) $\log w(\alpha)$ is concave.

(c) For some $\alpha_{0}$ and real $A_{0}, w(\alpha) \geq e^{-A_{0} \alpha}$ for $\alpha \geq \alpha_{0}$.

Lemma A.1. Let $w$ be a weight. Then:

(i)

$$
w(\alpha+\beta) \leq w(\alpha) w(\beta)
$$

(ii) $1 / \alpha \log w(\alpha)$ is monotone decreasing

(iii) $A(w) \equiv-\inf _{\alpha} 1 / \alpha \log w(\alpha)=-\lim _{\alpha \rightarrow \infty} 1 / \alpha \log w(\alpha)<\infty$

(iv) $w(\alpha) e^{A(w) \alpha}$ is monotone increasing; in particular,

$$
w(\alpha) \geq e^{-A(w) \alpha}
$$

for all $\alpha$.

Proof. (i) By (a) and (b), $w(\alpha) \geq w(\alpha+\beta)^{\alpha / \alpha+\beta}$ and $w(\beta) \geq w(\alpha+$ $\beta)^{\beta / \alpha+\beta}$. (A.1) results by taking the product of these relations.

(ii) If $\alpha<\beta$, then by (a) and (b),

$$
w(\alpha) \geq w(\beta)^{\alpha / \beta} \text {. }
$$

(iii) is a consequence of (c).

(iv) Given $\alpha<\beta<\gamma$, we have by (b) that

$$
w(\beta) \geq w(\alpha)^{(\gamma-\beta) /(\gamma-\alpha)} w(\gamma)^{(\beta-\alpha) /(\gamma-\alpha)} .
$$

By (iii), $\lim _{\gamma \rightarrow \infty} w(\gamma)^{1 / \gamma}=e^{-A(w)}$ so as $\gamma \rightarrow \infty$, we have that

$$
w(\beta) \geq w(\alpha) e^{-A(\beta-\alpha)},
$$

which is the required monotonicity. The final statement is just $w(\alpha) e^{A(w) \alpha} \geq w(0) e^{A(w) 0}$.

Remarks. 1. For many facts below, only (A.1) is critical, but we will need (A.3) in one place, so requiring log concavity seemed reasonable.

2. The prime example of weights is

$$
w_{B, \ell}(\alpha)=(1+\alpha)^{\ell} e^{-B \alpha}
$$

for any $B \in \mathbb{R}$ and $\ell \geq 0$. Notice that $A\left(w_{A, \ell}\right)=B$.

Definition. Let $n$ be a non-negative integer and $w$ a weight. We define $C_{n, w}$ to be those complex valued functions $f \in L^{1}([0, \infty), w(\alpha) d \alpha)$ with $n$ distributional derivatives (viewed as distributions on $C_{0}^{\infty}(0, \infty)$ ) $f^{\prime}, \ldots, f^{(n)}$ all in $L^{1}([0, \infty), w(\alpha) d \alpha)$ (so $C_{n=0, w}$ is $\left.L^{1}(w d \alpha)\right)$. If $f \in$ 
$C_{n, w}$ with $n \geq 1$, then $f$ is $C^{n-1}$ in classical sense and $\lim _{\alpha \downarrow 0} f^{(\ell)}(\alpha)$ exists for $\ell=0,1, \ldots, n-1$ which we denote by $f^{(\ell)}(0)$. We norm $C_{n, w}$ with

$$
\|f\|_{n, w}=\sum_{\ell=0}^{n-1}\left|f^{(\ell)}(0)\right|+\int_{0}^{\infty}\left[\sum_{\ell=0}^{n}\left|f^{(\ell)}(\alpha)\right|\right] w(\alpha) d \alpha .
$$

We denote $C_{n, w_{B, \ell}}$ by $C_{n}^{B, \ell}$ where $w_{B, \ell}$ is given by (A.4). As usual, the convolution of two $L_{\text {loc }}^{1}$ functions on $[0, \infty)$ is given by

$$
(f * g)(\alpha)=\int_{0}^{\alpha} f(\beta) g(\alpha-\beta) d \beta .
$$

By induction, it is easy to see that if $f, g \in C_{n, w}$, then for $1 \leq \ell \leq n$,

$$
(f * g)^{(\ell)}(\alpha)=\sum_{j=0}^{\ell-1} f^{(j)}(0) g^{(\ell-1-j)}(\alpha)+\left(f^{(\ell)} * g\right)(\alpha) .
$$

This formula and (A.1) imply that $C_{n, w} * C_{n, w} \subset C_{n, w}$ and, by (A.5)

$$
\|f * g\|_{n, w} \leq\|f\|_{n, w}\|g\|_{n, w}
$$

so that $C_{n, w}$ is a Banach algebra (without unit) under convolution.

Given $\mu \in \mathbb{C}$ and $f \in C_{n, w}$, we define the Laplace transform $L(\mu, f)(z)$ for $z \in D(A(w)) \equiv \infty \cup\{z \mid \operatorname{Re} z \geq A(w)\}$ by

$$
\begin{aligned}
L(\mu, f)(z) & =\mu+\int_{0}^{\infty} f(\alpha) e^{-\alpha z} d \alpha & & (z \neq \infty) \\
& =\mu & & (z=\infty) .
\end{aligned}
$$

By (A.2), the integral in (A.7) converges absolutely and defines a function analytic in $D(A(w))^{\text {int }}$ and continuous on $D(A(w))$. We denote the set of functions $F(z)=L(\mu, f)(z)$ for some $f \in C_{n, w}$ by $\mathfrak{A}_{n, w}$. For $w=w_{B, \ell}$, we denote $\mathfrak{A}_{n, w}$ by $\mathfrak{A}_{n}^{B, \ell}$. We norm $\mathfrak{A}_{n, w}$ via $\left\||| L(\mu, f)\left|\left\|_{n, w}=|\mu|+\right\| f \|_{n, w}\right.\right.$. Since

$$
L(\mu, f) L(\lambda, g)=L(\mu \lambda, \mu g+\lambda f+f * g),
$$

(A.6) shows that $\mathfrak{A}_{n, w}$ is a Banach algebra with unit.

We will need the following result of Wiener type:

Theorem A.2. Suppose $F \in \mathfrak{A}_{n, w}$ obeys $F(z) \neq 0$ for all $z$ in $D(A(w))$. Then $G(z) \equiv 1 / F(z)$ is also in $\mathfrak{A}_{n, w}$.

Results of Wiener type are usually proven via the Gelfand theory of commutative Banach algebras (see [1], Ch. III for a good exposition). Using those ideas, Theorem A.2 is a direct consequence of 
Proposition A.3. Every multiplicative linear functional on $\mathfrak{A}_{n, w}$ is of the form $F \mapsto F(z)$ for $z$ fixed in $D(A(w))$.

Proof. Let $\varphi: \mathfrak{A}_{n, w} \rightarrow \mathbb{C}$ be a multiplicative linear functional. Since $L(1,0)$ is the identity, one has $\varphi(L(1,0))=1$, so $\varphi$ is determined by the functional $T(f)=\varphi(L(0, f))$ on the space $C_{n, w}$. We claim that $T$ is determined by its values on $C_{0}^{\infty}(0, \infty) \subset C_{n, w}$. If $n=0$, this is obvious since $C_{0}^{\infty}(0, \infty)$ is then dense in $C_{n, w}$. But if $n>0, \overline{C_{0}^{\infty}(0, \infty)}$ is not all of $C_{n, w}$, so the argument is more subtle.

Indeed, if $n \geq 1$, any $f$ in $C_{n, w}$ is $C^{n-1}$ in the classical sense and $f \in \overline{C_{0}^{\infty}(0, \infty)}$ has $f(0)=f^{\prime}(0)=\cdots=f^{(n-1)}(0)=0$. In fact, it is not hard to see that $\overline{C_{0}^{\infty}(0, \infty)}$ has codimension $n-1$ in $C_{n, w}$.

Now for any $g \in C_{n, w}, g^{n+1}(n+1$-fold convolution) vanishes at 0 with its first $n-1$ derivative zero, and so it lies in $\overline{C_{0}^{\infty}(0, \infty)}$. If $T\left(g^{n+1}\right)=0$, then $T(g)=0$, and if $T\left(g^{n+1}\right) \neq 0$, then $T(g)=T\left(g^{n+2}\right) / T\left(g^{n+1}\right)$. Either way, $T(g)$ is determined by $T$ on $C_{0}^{\infty}(0, \infty)$.

The map $g \mapsto T(g)$ for $g \in C_{0}^{\infty}(0, \infty)$ defines a distribution which we denote by $T(\alpha)$. Formally, $T(f)=\int_{0}^{\infty} T(\alpha) f(\alpha) d \alpha$ and

$$
T(\alpha+\beta)(f(\alpha) g(\beta))=\int_{0}^{\infty} \int_{0}^{\infty} d \alpha d \beta T(\alpha+\beta) f(\alpha) g(\beta) .
$$

The idea of the proof is to derive the functional equation (A.8) for $T(\alpha)$ and to show that the distribution $T(\alpha)$ is in fact a regular distribution corresponding to a smooth function. If this is done, then one derives from (A.8) that $T(\alpha)$ is an exponential. If this is done, the proof is easy to complete.

The convolution formula for the Laplace transform implies $T(f * g)=$ $T(f) T(g)$. Since $T(\alpha+\beta)(f(\alpha) g(\beta))=T(f) T(g)$ and, since the linear span of the set of products of functions $f(\alpha) g(\beta)$ is dense in the set of functions $C_{0}^{\infty}\left(R_{+} \times R_{+}\right)$where $R_{+}:=(0, \infty)$, one concludes that

$$
T(\alpha+\beta)=T(\alpha) T(\beta)
$$

in distributional sense. If $T \not \equiv 0$, then $T(f) \neq 0$ for some $f \in C_{0}^{\infty}$. Thus,

$$
T(\beta)=\int_{0}^{\infty} T(\alpha+\beta) f(\alpha) d \alpha / T(f)
$$

so $T$ is a $C^{\infty}$ function. Thus, (A.8) implies that $T^{\prime}(\alpha) / T(\alpha)$ is constant and then that $T(\alpha)=e^{-z \alpha}$ for some $z \in \mathbb{C}$ (or else $T \equiv 0$ ).

Suppose that Re $z<A(w)$. Then $w(\alpha) \leq C_{1} \exp \left(-\frac{1}{2}(A(w)+\operatorname{Re} z) \alpha\right)$ and thus, $f(\alpha) \equiv \alpha^{n} e^{z \alpha}$ lies in $C_{n, w}$. But if $\eta \in C_{0}^{\infty}(0, \infty)$, then $T(\eta f)=\int_{0}^{\infty} T(\alpha) \eta(\alpha) e^{z \alpha} \alpha^{n} d \alpha=\int_{0}^{\infty} \alpha^{n} \eta(\alpha) d \alpha$. Taking $\eta$ a smoothed out characteristic function of $\left(\delta, \delta^{-1}\right)$, and taking $\delta \rightarrow 0$, has $\eta_{\delta} f \rightarrow f$ 
in $C_{n, w}$ but $T\left(\eta_{\delta} f\right) \rightarrow \infty$. Thus, Re $z \geq A(w)$, that is, $z \in D(A(w))$. Thus every $\varphi$ is an evaluation at some $z \in D(A(w))$. Every such evaluation clearly defines a multiplicative linear functional.

We also need to know about factoring out zeros.

Theorem A.4. Let $z_{0} \in D(A(w))^{\text {int }}$ and let $F \in \mathfrak{A}_{n, w}$ obey $F\left(z_{0}\right)=0$. Then $G(z)=F(z) /\left(z-z_{0}\right)$ lies in $\mathfrak{A}_{n+1, w}$.

Proof. Suppose first that $F(\infty)=0$ so $F=L(0, f)$ for some $f$ in $C_{n, w}$. Let $\tilde{A}=\operatorname{Re} z_{0}+1>A(w)$ so $f \in C_{n}^{\tilde{A}}$. Let $H(z)=\left(z-z_{0}\right)^{-1}=L(0, h)$, where

$$
h(\alpha)=e^{\alpha z_{0}}
$$

so $h \in C_{n}^{\tilde{A}, \ell=0}$. Thus in $D(\tilde{A}), G(z)=L(0, g)$ with $g=h * f$, that is,

$$
\begin{aligned}
g(\alpha) & =\int_{0}^{\alpha} h(\alpha-\beta) f(\beta) d \beta \\
& =\int_{0}^{\alpha} e^{(\alpha-\beta) z_{0}} f(\beta) d \rho \\
& =-\int_{\alpha}^{\infty} e^{(\alpha-\beta) z_{0}} f(\beta) d \beta
\end{aligned}
$$

since $F\left(z_{0}\right)=0$ means $\int_{0}^{\infty} e^{-\beta z_{0}} f(\beta) d \beta=0$.

Thus,

$$
\begin{aligned}
\int_{0}^{\infty}|g(\alpha)| w(\alpha) d \alpha & \leq \int_{0}^{\alpha}\left(\int_{\alpha}^{\infty} e^{(\alpha-\beta) \operatorname{Re} z_{0}}|f(\beta)| d \beta\right) w(\alpha) d \alpha \\
& =\int_{0}^{\infty}\left(\int_{0}^{\beta} e^{(\alpha-\beta) \operatorname{Re} z_{0}} w(\alpha) d \alpha\right)|f(\beta)| d \beta
\end{aligned}
$$

By Lemma A.1(iv) for $\alpha \leq \beta$ :

$$
\begin{aligned}
e^{(\alpha-\beta) \operatorname{Re} z_{0}} w(\alpha) & \leq e^{(\alpha-\beta) \operatorname{Re} z_{0}} e^{-A(w)(\alpha-\beta)} w(\beta) \\
& =e^{-(\alpha-\beta)\left(A(w)-\operatorname{Re} z_{0}\right)} w(\beta) .
\end{aligned}
$$

Since $A(w) \operatorname{Re} z_{0}<0$,

$$
\int_{0}^{\beta} e^{-(\alpha-\beta)\left(A-\operatorname{Re} z_{0}\right)} d \alpha=\int_{0}^{\beta} e^{\left(A-\operatorname{Re} z_{0}\right) \gamma} d \gamma
$$

is bounded by $-\left(A-\operatorname{Re} z_{0}\right)^{-1}$ independent of $\beta$ so (A.11) shows that

$$
\int_{0}^{\beta}|g(\alpha)| w(\alpha) d \alpha \leq\left(\operatorname{Re} z_{0}-A\right)^{-1} \int_{0}^{\alpha}|f(\beta)| w(\beta) d \beta
$$

and thus $g \in L^{1}(w(\alpha) d \alpha)$. 
By (A.6) for $1 \leq \ell \leq n+1$,

$$
g^{(\ell)}(\alpha)=\sum_{j=0}^{\ell-1} z_{0}^{j} f^{(\ell-1-j)}(\alpha)+z_{0}^{\ell} g(\alpha) .
$$

(A.13) and (A.14) therefore show that $g \in C_{n+1, w}$ as required.

For general $F$ with $F(\infty)=\mu$, pick $z_{1}$ with $\operatorname{Re} z_{1}<A(w)$ and define $F_{0}$ by

$$
F(z)=\mu \frac{z-z_{0}}{z-z_{1}}+F_{0}(z)
$$

Since

$$
\frac{z-z_{0}}{z-z_{1}}=1+\left(z_{0}-z\right) \int_{0}^{\infty} e^{\alpha z_{1}} e^{-\alpha z} d \alpha
$$

and $e^{\cdot z_{1}} \in C_{n, w}$ for all $n$, we see $F_{0}(z) \in \mathfrak{A}_{n, w}$ with $F_{0}(\infty)=F_{0}\left(z_{0}\right)=0$. Thus, $G(z)=\mu\left(z-z_{1}\right)^{-1}+G_{0}(z)$ lies in $\mathfrak{A}_{n, w}$.

This theorem has an interesting corollary (immediate consequence of Theorem A.2):

Corollary A.5. Let $F \in \mathfrak{A}_{n, w}$ be non-vanishing on $D(A(w))$ except for zeros at $z_{1}, \ldots, z_{m} \in D(A(w))$ int of order $\ell_{1}, \ldots, \ell_{m}$. Then for any $z_{0} \notin D(A(w))$,

$$
F(z)=G(z) \prod_{j=1}^{m}\left(\frac{z-z_{j}}{z-z_{0}}\right)^{\ell_{j}},
$$

where $G$ is non-vanishing on all of $D(A(w))$ and lies in $\mathfrak{A}_{k, w}$. In particular, $G$ is invertible, and any two such $F$ 's generate the same principal ideal.

We will need to know what happens where $z_{0} \in \partial D(A(w))$. First, we will consider $z_{0} \neq \infty$ when we lose some decay of the inverse Laplace transform.

Theorem A.6. Let $w$ be a weight and define the weight

$$
\tilde{w}(\alpha)=(1+|\alpha|) w(\alpha) .
$$

Let $\operatorname{Re} z_{0}=A(w)$ and let $F \in \mathfrak{A}_{n, \tilde{w}}$ obey $F\left(z_{0}\right)=0$. Then $G(z)=$ $F(z) /\left(z-z_{0}\right)$ lies in $\mathfrak{A}_{n+1, w}$.

Remark. Notice that $F \in \mathfrak{A}_{n, \tilde{w}}$ but $G \in \mathfrak{A}_{n-1, w}$ with $w$, not $\tilde{w}$, so we lose one degree of decay for $L^{-1}(F)$. For $w_{B, \ell}$, this is not surprising. Since $F$ is analytic in $D(A(w))^{\text {int }}$, dividing by $\left(z-z_{0}\right)$ does not lose 
smoothness of $F$ but since $F$ is only $C^{\ell}$ on $\partial D(A(w))$, we expect the division to lose one order of smoothness.

Proof. As in the proof of Theorem A.4, (A.15) lets us consider the case $F(\infty)=0$ and (A.14) lets us reduce to the case $n=0$. In that case, one follows the proof of Theorem A.4, but since $-A(w)=\operatorname{Re} z_{0}$, (A.12) becomes

$$
\int_{0}^{\beta} d \alpha=\beta
$$

so (A.13) becomes

$$
\begin{aligned}
\int_{0}^{\infty}|g(\alpha)| w(\alpha) d \alpha & \leq \int_{0}^{\infty}|f(\beta)| \beta w(\beta) d \beta \\
& \leq \int_{0}^{\infty}|f(\beta)| \tilde{w}(\beta) d \beta .
\end{aligned}
$$

Finally, we need the case when $z_{0}=\infty$ where we need to trade off some smoothness of $L^{-1}(F)$ to "divide out" a single zero of $F$ at infinity.

Theorem A.7. Let $F \in \mathfrak{A}_{n, w}$ with $n \geq 1$ and suppose that $F(\infty)=0$. Then $G(z)=z F(z)$ lies in $\mathfrak{A}_{n-1, w}$.

Proof. By an integration by parts, if $\alpha \in D(A(w))$ and $f \in C_{n, w}$, then

$$
z \int_{0}^{\infty} e^{\alpha z} f(\alpha) d \alpha=f(0)-\int_{0}^{\infty} e^{\alpha z} f^{\prime}(\alpha) d \alpha .
$$

The final result we need is a simple consequence of the earlier theorems. Note that if $w$ is a weight and $\tilde{w}$ is given by (A.16) and $F \in \mathfrak{A}_{n, \tilde{w}}$, then $F$ is $C^{1}$ on the finite part of $\partial D(A(w))$. So it makes sense to talk about a simple zero (i.e., $F\left(z_{0}\right)=0, F^{\prime}\left(z_{0}\right) \neq 0$ ) for $z_{0} \in \partial D(A(w)$ ).

Theorem A.8. Let $F \in \mathfrak{A}_{n, w}$ with $n \geq 1$. Suppose that the only zeros of $F$ on $D(A(w))$ are at $z_{1}, \ldots, z_{k}$ all in $D(A(w))^{\text {int }}$ and are simple. Then there is $G \in \mathfrak{A}_{n, w}$ and $a_{1}, \ldots, a_{k} \in \mathbb{C}$ so that

$$
F(z)^{-1}=\sum_{\ell=1}^{k} \frac{a_{\ell}}{z-z_{\ell}}+G(z) .
$$

Equivalently for $z>\max \left(\operatorname{Re}\left(z_{\ell}\right)\right)$,

$$
F(z)^{-1}=F(\infty)^{-1}+\int_{0}^{\infty} e^{-\alpha z} h(\alpha) d \alpha,
$$


where

$$
h(\alpha)=-\sum_{\ell=1}^{k} a_{\ell} e^{\alpha z_{\ell}}+g(\alpha),
$$

where $g \in C_{n, w}$. The result remains true if a single $z_{\ell}$ has $\operatorname{Re} z_{\ell}=A(w)$ so long as $F \in \mathfrak{A}_{n, \tilde{w}}$ with $\tilde{\tilde{w}}$ given by

$$
\tilde{\tilde{w}}(\alpha)=(1+|\alpha|)^{2} w(\alpha) .
$$

We will need

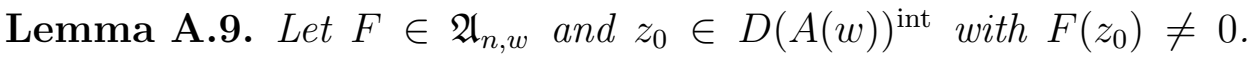
Then

$$
\frac{F(z)}{z-z_{0}}=\frac{F\left(z_{0}\right)}{z-z_{0}}+G(z)
$$

with $G \in \mathfrak{A}_{n+1, w}$. If $\operatorname{Re} z_{0}=A(w)$ and $F \in \mathfrak{A}_{n, \tilde{w}}$ with $\tilde{w}$ given by (A.16), then $G \in \mathfrak{A}_{n+1, w}$.

Proof of Lemma A.9. Write $F(z)=H(z)+F\left(z_{0}\right)$, where $H\left(z_{0}\right)=0$ and use Theorems A.4 and A.6.

Proof of Theorem A.8. By repeated use of Theorem A.4 and Corollary A.5, we can write

$$
F(z)=H(z) \prod_{j=1}^{m}\left(\frac{z-z_{j}}{z-z_{0}}\right),
$$

where $H$ is everywhere non-vanishing (it is because of the $z-z_{0}$ factors that $G(\infty) \neq 0)$. If all $z_{j}$ lie in $D(A(w))^{\text {int }}$, then $H \in \mathfrak{A}_{n+1, w}$. If one $z_{j}$ is in $D(A(w))$ and $F \in \mathfrak{A}_{n, \tilde{w}}$, then $H \in \mathfrak{A}_{n, \tilde{\tilde{w}}}$.

As a result,

$$
F(z)^{-1}=H(z)^{-1} \prod_{j=1}^{m}\left[1+\frac{z_{0}-z_{j}}{z-z_{j}}\right],
$$

where $H(z)^{-1}$ is in $\mathfrak{A}_{n, w}$ (resp. by $\mathfrak{A}_{n, \tilde{w}}$ ) by Theorem A.2.

By Lemma A.9,

$$
\left(1+\frac{z_{0}-z_{1}}{z-z_{1}}\right) H(z)=\frac{c_{1}}{z-z_{1}}+H_{1}(z),
$$

where $H_{1} \in \mathfrak{A}_{n, w}\left(\operatorname{resp} . \mathfrak{A}_{n, \tilde{w}}\right)$. Since

$$
\left(1+\frac{d_{1}}{z-z_{2}}\right)\left(\frac{c_{1}}{z-z_{1}}\right)=\frac{c_{2}}{z-z_{1}}+\frac{d_{2}}{z-z_{2}}
$$

for suitable $c_{2}, d_{2}$, we obtain the result inductively. 


\section{REFERENCES}

[1] I. Gelfand, D. Raikov, and G. Shilov, Commutative Normed Rings, Chelsea, New York, 1964.

[2] F. Gesztesy and B. Simon, A new approach to inverse spectral theory, II. General real potentials and the connection to the spectral measure, preprint.

[3] B. Ya. Levin, Fourier- and Laplace-type transformations by means of solutions of a second-order differential equation, Dokl. Akad. Nauk SSSR 106 (1956), 187-190 (Russian).

[4] V.A. Marchenko, Reconstruction of the potential energy from the phases of the scattered waves, Dokl. Akad. Nauk SSSR 104 (1955), 695-698 (Russian).

[5] V.A. Marchenko, Sturm-Liouville Operators and Applications, Birkhäuser, Basel-Boston-Stuttgart, 1986.

[6] A.G. Ramm, Recovery of the potential from I-function, C. R. Math. Rep. Acad. Sci. Canada IX (1987), 177-182.

[7] A.G. Ramm, Property $C$ for ODE and applications to inverse problems, Proceedings of the International Conference on Operator Theory, to appear.

[8] A.G. Ramm, Multidimensional Inverse Scattering Problems, Longman, New York, 1992.

[9] A.G. Ramm and B.A. Taylor, Example of a potential in one-dimensional scattering problem for which there are infinitely many purely imaginary resonances, Phys. Lett. A124 (1987), 313-319.

[10] B. Simon, A new approach to inverse spectral theory, I. Fundamental formalism, Ann. of Math., to appear.

[11] M. Zworski, Distribution of poles for scattering on the real line, J. Funct. Anal. 73 (1987), 277-296. 